\title{
ĐÁNH GIÁ TÁC ĐộNG CỦA VIỆC SỬ DỤNG THAM SỐ HÓA ĐỐI LƯU TRONG DỬ BÁO ĐỢT MƯA LỚN THÁNG 7 NĂM 2015 TRÊN KHU VỬC BẮC Bộ BẰNG MÔ HİNH PHÂN GIẢI CAO
}

\author{
Dư Đức Tiến ${ }^{1}$, Hoàng Đức Cường ${ }^{1}$, Mai Khánh Hưng ${ }^{1}$, Hoàng Phúc Lâm ${ }^{1}$
}

Tóm tắt: Bài báo trình bày thử nghiệm dụ báo đọt mưa lón kỉ luc trên khu vục Bắc Bộ tháng 7 năm 2015 bằng mô hình WRF-ARW với điều kiện biên tù̀ mô hình toàn cầu GFS (Mỹ). Việc hạ quy mô thực hiện thông qua thiết lập hai lưới tính $15 \mathrm{~km}$ và $5 \mathrm{~km}$ cho mô hình WRF-ARW và dư báo đến hạn 72h. Hai lớp thư nghiệm đã được thưc hiện gồm có sử dụng (CPS) và không sủ dụng tham số hóa đối lưu (noCPS) trên lưới tính phân giải cao $5 \mathrm{~km}$. Úng với mồi dụ báo, 32 trường hơp gồm các cấu hình khác nhau được thiết lập để loại trù trường hợp kết quả phu thuộc vào một cấu hình vật lý cu thể của mô hình WRF-ARW. Các kết quả đánh giá cho thấy ở hạn dụ báo 24 h tại các ngữong mưa lơn $(50 \mathrm{~mm} / 24 \mathrm{~h}$ và $100 \mathrm{~mm} / 24 \mathrm{~h})$ việc sủ dụng các so đồ tham số hóa (CPS) cho kết quả tốt hơn so với việc không sư dụng tham số hóa đối lư (noCPS) trên luới tính phân giải cao $5 \mathrm{~km}$. Tuy nhiên ở các hạn $48 \mathrm{~h}$ và $72 \mathrm{~h}$, khi không sư dụng tham số hóa đối lưu hay quá trình đối lưu được giải một cách tuờng mình trong mô hình (explicit) cho phép tăng được kĩ năng dụ báo hơn khi thử nghiệm dụ báo mua lón cho đợt mura lón trên khu vưc Bắc Bộ.

Từ khóa: Dự báo mua lớn Bắc Bộ, mô hình WRF-ARW, tham số hóa vật lý đối lưu.

Ban Biên tập nhận bài: 08/12/2018 Ngày phản biện xong: 27/01/2019 Ngày đăng bài: 25/03/2019

\section{Mở đầu}

Phương pháp số trị (NWP- Numerical Weather Prediction) hoặc phương pháp động lực sử dụng phương pháp số giải xấp xỉ các phương trình toán, lý mô phỏng các quá trình chuyển động trong khí quyển (hệ phương trình nhiệt động lực Navie-Stoke). Với năng lực tính toán được phát triển vượt bậc trong 5 - 10 năm trở lại đây đã cho phép hạ quy mô tính toán xuống quy mô đối lưu (convective scale) với độ phân giải ngang từ $500 \mathrm{~m}$ đến $2 \mathrm{~km}$ (các mô hình ở độ phân giải này còn được gọi là các mô hình không sử dụng tham số hóa đối lưu). Trong các mô hình này, các sơ đồ tham số hóa đối lưu được loại bỏ bằng việc bổ sung các phương trình bảo toàn ẩm cho các biến giáng thủy ở dạng rắn, lỏng... và do đó cho phép tính toán được cả các quá trình bình lưu đối với các dạng giáng thủy này [3]. Khi đó các biến dự báo mưa gần như được xem là

${ }^{1}$ Trung tâm Dư báo khi tượng thủy văn quốc gia

Email:duductien@gmail.com biến chuẩn đoán (prognostics). Mặc dù về mặt lý thuyết và thực hành, việc giải hiện (explicit) được các biến dự báo mưa sẽ có tính ưu việt hơn so với việc tham số hóa các quá trình dưới lưới (sub-grid) mà mô hình có thể mô phỏng/dự báo được, tuy nhiên vẫn nhiều công trình cho thấy các tham số hóa đặc biệt là tham số hóa đối lưu vẫn có vai trò và hiệu quả nhất định ngay cả ở độ phân giải dưới 5 km [4].

Với vấn đề nêu trên, nghiên cứu sẽ trình bày thử nghiệm việc có và không có sử dụng tham số hóa đối lưu trên lưới tính phân giải cao $(5 \mathrm{~km})$ bằng mô hình WRF-ARW (Mỹ) trong bài toán dự báo mưa lớn cho đợt mưa lớn trên khu vực Bắc Bộ. Mục 2 của bài báo là thiết kế thử nghiệm bao gồm giới thiệu mô hình dự báo, số liệu điều kiện biên, trường hợp thử nghiệm, số liệu quan trắc và phương pháp đánh giá. Những phân tích kết quả được đưa ra trong phần 3 của bài báo và 
một số kết luận chính được tổng kết trong phần 4.

\section{Thiết kế thí nghiệm}

\subsection{Mô hình số trị khu vỵc WRF-ARW}

Trong nghiên cứu sử dụng hệ thống mô hình khu vực WRF với nhân động lực ARW phiên bản 3.9.1.1 do Trung tâm dự báo môi trường quốc gia Mỹ $(N C E P)$ phát triển (goi tắt là WRF$A R W)$. Đây là hệ thống mô hình khu vực được áp dụng trong nghiên cứu và nghiệp vụ với các ứng dụng đa dạng từ mô phỏng lý tưởng xoáy, sóng núi đến áp dụng các bài toán dự báo thời tiết hoặc các điều kiện thời tiết nguy hiểm như mưa, bão/xoáy thuận nhiệt đới và được cộng đồng khoa học hỗ trợ và phát triển. Mô hình WRF-ARW cũng được thử nghiệm và áp dụng trong nghiệp vụ tại Việt Nam trong các lĩnh vực dự báo thời tiết và dự báo bão. Chi tiết hơn về mô hình WRF-ARW có thể tham khảo trong [5]. Một trong những đặc tính quan trọng của hệ thống WRF-ARW là cung cấp một số lượng tùy chọn các thuật toán sai phân, các sơ đồ vật lý và các phương pháp đồng hóa số liệu được phát triển bởi cộng đồng khoa học có uy tín, cho phép các nhà ứng dụng lựa chọn được các tùy biến phù hợp với khu vực địa phương và đối tượng cần nghiên cứu. Mô hình WRF-ARW được nghiên cứu và áp dụng phổ biến tại Việt Nam những năm vừa qua, các công trình điển hình có thể kể đến gồm trong nghiên cứu dự báo thời tiết và bão [1] hay trong việc áp dụng và dự báo tổ hợp [2].

Thử nghiệm thiết lập hai lưới tính $15 \mathrm{~km}$ và $5 \mathrm{~km}$ cho mô hình WRF-ARW, số 41 mực thẳng đứng và dự báo đến hạn $72 \mathrm{~h}$, minh họa 2 miền tính được đưa ra trong hình 1 . Lựa chọn vật lý cho mô hình WRF-ARW dựa trên việc thay đổi gồm (a) sơ đồ Kain-Fritsch (KF) hoặc BettsMiller-Janjic (BMJ) cho tham số hóa đối lưu (cu- mulus parameterization scheme-CPS); (b) sơ đồ bức xạ sóng ngăn s Goddard hoặc Dudhia; (d) sơ đồ tham số hóa lớp biên của Yonsei University (YSU) hoặc Mellor-Yamada-Janjic (MYJ) và (e) các sơ đồ vi vật lý mây từ đơn giản đến phức tạp gồm sơ đồ Lin, WSM3, WSM5 đến WSM6 (khép kín 6 bậc). Dựa trên việc tổ hợp các lựa chọn này có thể tạo ra tối đa gồm 32 cấu hình vật lý khác nhau và được chi tiết trong bảng 1 . Lưu ý thêm ở đây đối với sơ đồ lớp biên MYJ thì sơ đồ rối bế mặt sẽ được đặt là sơ đồ Janjic Eta so với sơ đồ khép kín rồi dựa trên giả thiết của Monin-Obukhov.

Như vậy, ứng với 32 cấu hình khác nhau sẽ có 2 thử nghiệm được thiết lập gồm giữ sơ đồ tham số hóa đối lưu ở cả hai miền tính $15 \mathrm{~km}$ và $5 \mathrm{~km}$, kí hiệu là lớp thử nghiệm CPS. Thử nghiệm thứ hai gồm việc chỉ giữ tham số hóa đối lưu ở miền tính $15 \mathrm{~km}$ và tắt sơ đồ đối lưu ở miền tính $5 \mathrm{~km}$, kí hiệu là lớp thử nghiệm này là noCPS. Khi đó ta sẽ khảo sát được ảnh hưởng của việc không sử dụng tham số hóa đối lưu ở độ phân giải cao mà vẫn xem xét được với các sơ đồ vật lý đa dạng khác nhau ở miền tính $15 \mathrm{~km}$. Úng với mỗi dự báo sẽ thực hiện 32 cấu hình vật lý (Bảng 1) và với hai lựa chọn có và không sử dụng CPS ở độ phân giải $5 \mathrm{~km}$, như vậy 1 ốp dự báo sẽ gồm 64 thử nghiệm dự báo.

\subsection{Số liệu điều kiện biên}

Nghiên cứu sử dụng dự báo từ mô hình toàn cầu GFS (Mỹ) làm điều kiện ban đầu và điều kiện biên cho mô hinh WRF-ARW, được cung cấp thông qua địa chỉ sau: http://www.nco.ncep.noaa.gov/pmb/products/gfs /. Số liệu GFS có độ phân giải ngang là $55 \mathrm{~km}$ với số mực áp suất thẳng đứng của mô hình GFS là 26 và được cập nhật 3 tiếng một cho mô hình WRF-ARW. 
BÀI BÁO KHOA HỌC

Bảng 1. Cấu hình vật lý và ký hiệu cho các thư nghiệm khác nhau cho mô hình WRF-RW

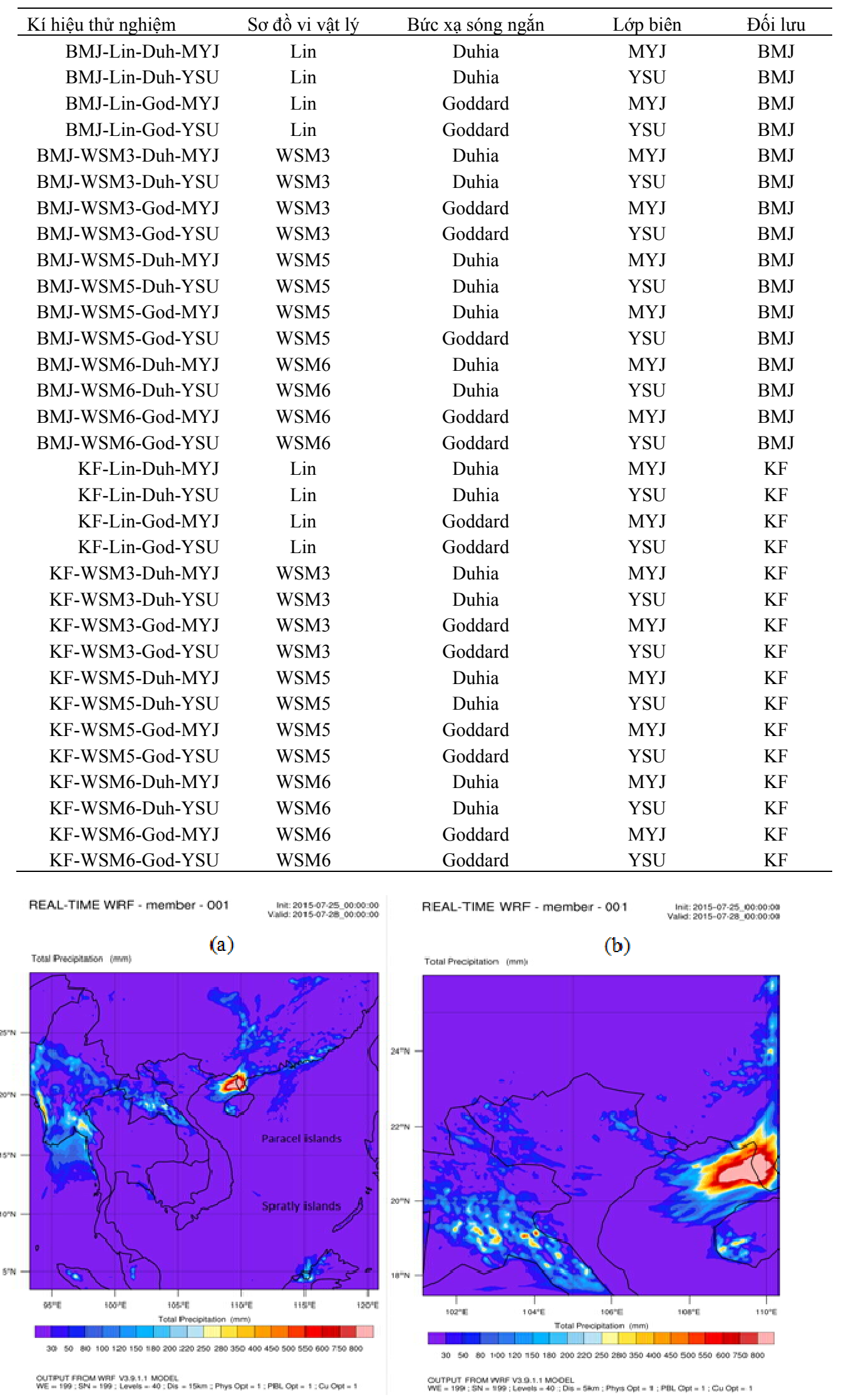

Hình 1. Minh họa 2 miền tính: (a) $15 \mathrm{~km}$ và (b) $5 \mathrm{~km}$ trong thử nghiệm dụ báo mưa lớn trên khu vục Bắc Bộ 


\subsection{Số liệu quan trắc}

Thử nghiệm thực hiện dự báo liên quan đến đợt mưa lớn kỉ lục trên khu vực Bắc Bộ do sự hoạt động của rãnh thấp trên cao kết hợp với vùng thấp tồn tại trên Vịnh Bắc Bộ vào tháng 7 năm 2015 từ ngày 25/7/2015 đến $01 / 8 / 2015$ và thực hiện dự báo tại các giờ $00 \mathrm{z}$. Chi tiết số liệu quan trắc tại các trạm sử dụng trong đánh giá trên khu vực Bắc Bộ được đưa ra trong Bảng 2.

Bảng 2. Danh sách các trạm trên khu vực Bắc Bộ để đánh giá kết quả dụ báo

\begin{tabular}{|c|c|c|c|c|c|c|c|c|c|}
\hline $\begin{array}{c}\text { Mã } \\
\text { trạm }\end{array}$ & Tên trạm & $\begin{array}{c}\text { Mã } \\
\text { trạm }\end{array}$ & Tên trạm & Mã trạm & $\begin{array}{c}\text { Tên } \\
\text { Trạm }\end{array}$ & Mã trạm & Tên Trạm & Mã trạm & Tên Trạm \\
\hline $48 / 01$ & Muong Te & 48800 & Lai Chau & $48 / 59$ & Chi Linh & $48 / 29$ & Pho Rang & 48820 & Ha Noi \\
\hline $48 / 02$ & Sin Ho & 48802 & $\mathrm{Sa} \mathrm{Pa}$ & $48 / 60$ & Uong Bi & $48 / 30$ & $\mathrm{Bac} \mathrm{Ha}$ & 48821 & Phu Ly \\
\hline $48 / 03$ & Tam Duong & 48803 & Lao Cai & $48 / 61$ & Kim Boi & $48 / 31$ & Hoang Su Phi & 48822 & Hung Yen \\
\hline $48 / 05$ & Muong La & 48805 & Ha Giang & $48 / 63$ & Chi Ne & $48 / 32$ & $\mathrm{Bac} \mathrm{Me}$ & 48823 & Nam Dinh \\
\hline $48 / 06$ & Than Uyen & 48806 & Son $\mathrm{La}$ & $48 / 64$ & Lac Son & $48 / 33$ & Bao Lac & 48824 & Ninh Binh \\
\hline $48 / 07$ & Quynh Nhai & 48807 & That Khe & $48 / 65$ & $\begin{array}{c}\text { Cuc } \\
\text { Phuong }\end{array}$ & $48 / 34$ & Bac Quang & 48826 & Phu Lien \\
\hline $48 / 08$ & Mu Cang Chai & 48808 & Cao Bang & $48 / 67$ & Yen Dinh & $48 / 35$ & Luc Yen & 48827 & Hai Duong \\
\hline $48 / 09$ & Tuan Giao & 48809 & Bac Giang & $48 / 68$ & Sam Son & $48 / 36$ & Ham Yen & 48828 & Hon Dau \\
\hline $48 / 10$ & Pha Din & $48 / 81$ & Hon Ngu & $48 / 69$ & $\begin{array}{c}\text { Bai } \\
\text { Thuong }\end{array}$ & $48 / 37$ & Chiem Hoa & 48829 & Van Ly \\
\hline $48 / 14$ & Van Chan & 48810 & Bac Can & $48 / 70$ & $\begin{array}{l}\text { Nhu } \\
\text { Xuan }\end{array}$ & $48 / 39$ & Cho Ra & 48830 & Lang Son \\
\hline $48 / 16$ & Song Ma & 48811 & Dien Bien Phu & $48 / 72$ & Tinh Gia & $48 / 40$ & Nguyen Binh & 48831 & Thai Nguyen \\
\hline $48 / 17$ & Co Noi & 48812 & Tuyen Quang & $48 / 74$ & $\begin{array}{l}\text { Quy } \\
\text { Chau }\end{array}$ & $48 / 42$ & Ngan Son & 48832 & Nho Quan \\
\hline $48 / 18$ & Yen Chau & 48813 & Viet Tri & $48 / 75$ & Quy Hop & $48 / 43$ & Trung Khanh & 48833 & Bai Chay \\
\hline $48 / 19$ & Bac Yen & 48814 & Vinh Yen & $48 / 76$ & Tay Hieu & $48 / 44$ & Dinh Hoa & 48834 & Co To \\
\hline $48 / 20$ & Phu Yen & 48815 & Yen Bai & $48 / 77$ & $\begin{array}{l}\text { Quynh } \\
\text { Luu }\end{array}$ & $48 / 47$ & Bac Son & 48835 & Thai Binh \\
\hline $48 / 23$ & Minh Dai & 48817 & Son Tay & $48 / 79$ & $\begin{array}{c}\text { Con } \\
\text { Cuong }\end{array}$ & $48 / 48$ & Huu Lung & 48836 & Cua Ong \\
\hline $48 / 25$ & Moc Chau & 48818 & Hoa Binh & $48 / 80$ & $\begin{array}{l}\text { Do } \\
\text { Luong }\end{array}$ & $48 / 49$ & Dinh Lap & 48837 & Tien Yen \\
\hline $48 / 26$ & Mai Chau & $48 / 82$ & Huong Son & $48 / 51$ & Phu Ho & $48 / 50$ & Quang Ha & 48838 & Mong Cai \\
\hline
\end{tabular}

\subsection{Phuơng pháp đánh giá}

Để đánh giá kỹ năng dự báo xảy ra mưa lớn chúng tôi sử dụng chỉ số kĩ năng TS (Threat Score). Trong bảng 3 là bảng phân loại (Contingency table) theo hiện tượng dự báo (ở đây là mưa với các ngưỡng đánh giá cụ thể, ví dụ > $25 \mathrm{~mm} / 24 \mathrm{~h}$ tại từng trạm quan trắc).

Bảng 3. Bảng phân loại tần xuất cho biến dụ báo dạng nhị phân

\begin{tabular}{cccc}
\hline & & \multicolumn{3}{c}{ Quan trắc } \\
\cline { 3 - 4 } Dự báo & Có & Có & Không \\
& Không & A & B \\
\hline
\end{tabular}

Khi đó chỉ số kĩ năng:

$$
\mathrm{TS}=\frac{\mathrm{A}}{\mathrm{A}+\mathrm{B}+\mathrm{C}}
$$

TS có giá trị càng lớn (tiến đến 1 ) thì dự báo càng có kĩ năng, có giá trị thấp ứng với mô hình có kĩ năng thấp. Đây là chỉ số kĩ năng được sử dụng phổ biến khi đánh giá kết quả trên không gian trạm quan trắc. Mưa dự báo từ mô hình sẽ được nội suy theo phương pháp điểm lưới gần nhất với trạm để đưa kết quả về trạm quan trắc.

\section{3. Đánh giá kết quả}

Kết quả tính toán chỉ số kĩ năng TS tính cho 32 thử nghiệm trong đó đồng thời giữ các tham số hóa đối lưu (CPS) ở hai miền tính $15 \mathrm{~km}$ và $5 \mathrm{~km}$ và chỉ giữ ở miền tính $15 \mathrm{~km}$ được đưa ra chi tiết trong bảng 4 cho hai ngưỡng $25 \mathrm{~mm} / 24 \mathrm{~h}$ và $50 \mathrm{~mm} / 24 \mathrm{~h}$. Lưu ý rằng kết quả cuối cùng chỉ đánh giá trên miền tính $5 \mathrm{~km}$. Mẫu tính toán ra chỉ số TS ứng với mỗi trường hợp là 896. Trung 
bình giá trị kĩ năng TS ở hạn $24 \mathrm{~h}, 48 \mathrm{~h}$ và $72 \mathrm{~h}: \quad 50 \mathrm{~mm} / 24 \mathrm{~h} \sim 0.2$, ở ngưỡng cao hơn $100 \mathrm{~mm} / 24 \mathrm{~h}$ đối với ngưỡng $25 \mathrm{~mm} / 24 \mathrm{~h} \sim 0.33$, ngưỡng chỉ số kĩ năng giảm mạnh $\sim 0.1$.

Bảng 4. Chỉ số kĩ năng TS tính cho 32 thử nghiệm trong đó đồng thời giũu các tham số hóa đối lưu (CPS) ở hai miền tính $15 \mathrm{~km}$ và $5 \mathrm{~km}$ và chỉ giữ ở miền tính $15 \mathrm{~km}$ ở các hạn dụ báo $24 \mathrm{~h}, 48 \mathrm{~h}$ và $72 \mathrm{~h}$ cho hai ngương đánh giá $25 \mathrm{~m} / 24 \mathrm{~h}$ và $48 \mathrm{~mm} / 24 \mathrm{~h}$

\begin{tabular}{|c|c|c|c|c|c|c|c|c|c|c|c|c|}
\hline & \multicolumn{6}{|c|}{$25 \mathrm{~mm} / 24 \mathrm{~h}$} & \multicolumn{6}{|c|}{$50 \mathrm{~mm} / 24 \mathrm{~h}$} \\
\hline & CPS & noCPS & $\mathrm{CPS}$ & noCPS & CPS & noCPS & CPS & noCPS & CPS & noCPS & CPS & noCPS \\
\hline BMJ-Lin-Duh-MYJ & 0.19 & 0.28 & 0.22 & 0.25 & 0.28 & 0.27 & 0.09 & 0.12 & 0.12 & 0.19 & 0.17 & 0.19 \\
\hline BMJ-Lin-God-MYJ & 0.27 & 0.31 & 0.31 & 0.30 & 0.30 & 0.26 & 0.16 & 0.16 & 0.15 & 0.18 & 0.18 & 0.17 \\
\hline BMJ-Lin-God-YSU & 0.29 & 0.30 & 0.30 & 0.33 & 0.28 & 0.30 & 0.15 & 0.15 & 0.16 & 0.21 & 0.16 & 0.22 \\
\hline BMJ-WSM3-Duh-MYJ & 0.21 & 0.33 & 0.30 & 0.30 & 0.31 & 0.38 & 0.12 & 0.18 & 0.15 & 0.17 & 0.20 & 0.21 \\
\hline BMJ-WSM3-Duh-YSU & 0.24 & 0.26 & 0.29 & 0.35 & 0.33 & 0.34 & 0.12 & 0.15 & 0.15 & 0.22 & 0.20 & 0.19 \\
\hline BMJ-WSM5-Duh-YSU & 0.22 & 0.28 & 0.31 & 0.35 & 0.33 & 0.34 & 0.14 & 0.16 & 0.21 & 0.20 & 0.22 & 0.21 \\
\hline BMJ-WSM5-God-MYJ & 0.27 & 0.35 & 0.32 & 0.36 & 0.31 & 0.38 & 0.12 & 0.16 & 0.23 & 0.20 & 0.20 & 0.25 \\
\hline BMJ-WSM5-God-YSU & 0.30 & 0.34 & 0.30 & 0.34 & 0.29 & 0.34 & 0.16 & 0.17 & 0.22 & 0.21 & 0.16 & 0.24 \\
\hline BMJ-WSM6-Duh-MYJ & 0.22 & 0.35 & 0.28 & 0.30 & 0.25 & 0.33 & 0.16 & 0.19 & 0.15 & 0.22 & 0.16 & 0.19 \\
\hline BMJ-WSM6-Duh-YSU & 0.25 & 0.32 & 0.29 & 0.36 & 0.32 & 0.36 & 0.15 & 0.16 & 0.18 & 0.22 & 0.22 & 0.19 \\
\hline BMJ-WSM6-God-MYJ & 0.32 & 0.36 & 0.32 & 0.37 & 0.28 & 0.36 & 0.20 & 0.21 & 0.18 & 0.23 & 0.15 & 0.22 \\
\hline BMJ-WSM6-God-YSU & 0.30 & 0.37 & 0.36 & 0.34 & 0.26 & 0.35 & 0.21 & 0.22 & 0.20 & 0.17 & 0.14 & 0.26 \\
\hline KF-Lin-Duh-YSU & 0.38 & 0.33 & 0.31 & 0.25 & 0.30 & 0.30 & 0.22 & 0.17 & 0.18 & 0.16 & 0.18 & 0.20 \\
\hline KF-Lin-God-MYJ & 0.35 & 0.33 & 0.33 & 0.33 & 0.34 & 0.35 & 0.20 & 0.17 & 0.18 & 0.21 & 0.20 & 0.24 \\
\hline KF-Lin-God-YSU & 0.39 & 0.34 & 0.33 & 0.30 & 0.36 & 0.36 & 0.23 & 0.18 & 0.22 & 0.18 & 0.21 & 0.21 \\
\hline KF-WSM3-Duh-MYJ & 0.36 & 0.37 & 0.30 & 0.34 & 0.35 & 0.31 & 0.24 & 0.21 & 0.18 & 0.22 & 0.20 & 0.25 \\
\hline KF-WSM3-Duh-YSU & 0.38 & 0.35 & 0.37 & 0.34 & 0.37 & 0.33 & 0.24 & 0.17 & 0.24 & 0.21 & 0.28 & 0.24 \\
\hline KF-WSM3-God-MYJ & 0.43 & 0.38 & 0.30 & 0.33 & 0.34 & 0.29 & 0.28 & 0.21 & 0.20 & 0.22 & 0.17 & 0.18 \\
\hline KF-WSM3-God-YSU & 0.41 & 0.38 & 0.34 & 0.37 & 0.36 & 0.36 & 0.26 & 0.22 & 0.23 & 0.22 & 0.24 & 0.27 \\
\hline KF-WSM5-Duh-MYJ & 0.39 & 0.37 & 0.33 & 0.31 & 0.33 & 0.30 & 0.26 & 0.16 & 0.21 & 0.23 & 0.19 & 0.19 \\
\hline KF-WSM5-Duh-YSU & 0.39 & 0.35 & 0.38 & 0.30 & 0.36 & 0.34 & 0.22 & 0.20 & 0.25 & 0.25 & 0.26 & 0.24 \\
\hline KF-WSM5-God-MYJ & 0.43 & 0.37 & 0.32 & 0.39 & 0.34 & 0.29 & 0.29 & 0.22 & 0.21 & 0.29 & 0.18 & 0.21 \\
\hline KF-WSM5-God-YSU & 0.43 & 0.38 & 0.37 & 0.35 & 0.35 & 0.35 & 0.29 & 0.22 & 0.24 & 0.25 & 0.25 & 0.26 \\
\hline KF-WSM6-Duh-MYJ & 0.39 & 0.37 & 0.35 & 0.37 & 0.33 & 0.33 & 0.26 & 0.19 & 0.27 & 0.23 & 0.15 & 0.22 \\
\hline KF-WSM6-Duh-YSU & 0.41 & 0.35 & 0.37 & 0.37 & 0.36 & 0.33 & 0.28 & 0.19 & 0.30 & 0.23 & 0.26 & 0.23 \\
\hline KF-WSM6-God-MYJ & 0.43 & 0.38 & 0.36 & 0.36 & 0.33 & 0.37 & 0.31 & 0.24 & 0.26 & 0.25 & 0.17 & 0.21 \\
\hline KF-WSM6-God-YSU & 0.42 & 0.38 & 0.40 & 0.36 & 0.38 & 0.33 & 0.27 & 0.22 & 0.27 & 0.26 & 0.22 & 0.26 \\
\hline
\end{tabular}

Trong hình 2 minh họa dưới dạng biểu đồ cho chỉ số kĩ năng TS trên toàn bộ khu vực Bắc Bộ ở ngưỡng trên $25 \mathrm{~mm} / 24 \mathrm{~h}$ (hình $\mathrm{a}$ ), ngưỡng $50 \mathrm{~mm} / 24 \mathrm{~h}$ (hình b, ngưỡng $100 \mathrm{~mm} / 24 \mathrm{~h}$ (hình c) tại 3 hạn dự báo $24 \mathrm{~h}, 48 \mathrm{~h}$ và $72 \mathrm{~h}$ cho hai lớp thử nghiệm có dùng tham số hóa đối lưu ở miền tính
$5 \mathrm{~km}$ (CPS) và không sử dụng (noCPS). Đối với hạn dự báo 24h, các lớp thử nghiệm noCPS cho thấy kĩ năng hầu như thấp hơn CPS tại các ngưỡng mưa đánh giá khác nhau. Tại hạn dự báo dài hơn (48h và $72 \mathrm{~h}$ ), với ngưỡng mưa > $25 \mathrm{~mm} / 24 \mathrm{~h}$ sự khác biệt giữa $\mathrm{CPS}$ và noCPS là 
không đáng kể (hình 2.a). Tuy nhiên ứng với các hạn dự báo $48 \mathrm{~h}$ và $72 \mathrm{~h}$ này, ở ngưỡng mưa lớn hơn (50mm và $100 \mathrm{~mm})$, kĩ năng của noCPS tăng khá rõ rệt so với CPS. Kết quả này khá tương đồng với nghiên cứu của Gilliland và cộng sự [4] như đã đề cập đến trong phần 1 trong đó ở hạn dự báo ngắn, các sơ đồ tham số hóa đối lưu có thể giúp mô hình giảm thời gian thích ứng (spinup) qua đó có thể tăng chất lượng nắm bắt các hiện tượng đối lưu ở hạn ngắn, tuy nhiên ở hạn dự báo dài hơn, việc các hệ thống đối lưu có tổ chức quy mô vừa hay đối lưu sâu phát triển mạnh thì sử dụng mô hình quy mô đối lưu và không sử dụng tham số hóa sẽ có ưu thế rõ rệt.
Kết quả này có thể thấy rõ trong hình $2 \mathrm{~d}$ ứng với các giá trị trung bình TS được tích tách biệt cho các thử nghiệm sử dụng đối lưu BMJ (BMJMEAN) và sử dụng KF (KF-MEAN) tương ứng với hai lớp thử nghiệm CPS và noCPS. Bên cạnh đó, một đánh giá về trung bình tổ hợp đơn giản từ 32 dự báo khác nhau này cũng được thực hiện, kí hiệu là ENS-MEAN tại hình 2 cũng cho thấy rõ sự cải thiện khi không sử dụng CPS ở các ngưỡng mưa lớn và hạn sau $24 \mathrm{~h}$. Nếu so sánh riêng giữa $\mathrm{BMJ}$ và $\mathrm{KF}$ thì hầu hết việc không sử dụng CPS ở các lớp thử nghiệm BMJ đều giảm được sai số đi khá rõ. (a)

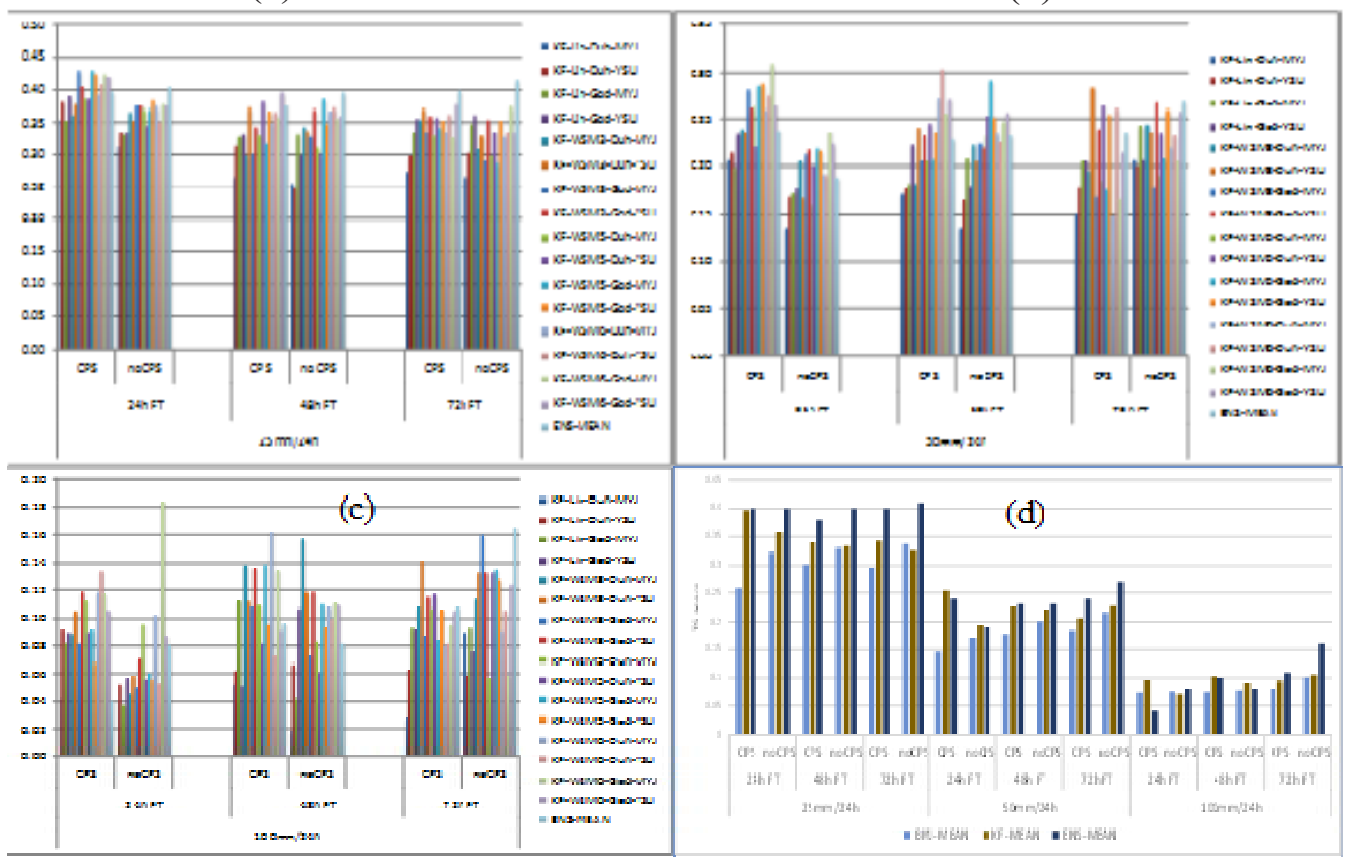

Hình 2. Biểu đồ minh họa chỉ số kĩ năng TS trên toàn bộ khu vực Bắc Bộ ở nguõng trên $25 \mathrm{~mm} / 24 \mathrm{~h}$ (a) nguõng $50 \mathrm{~mm} / 24 \mathrm{~h}$; (b) ngữ̛ng $100 \mathrm{~mm} / 24 \mathrm{~h}$; (c) tại 3 hạ dụ báo $24 \mathrm{~h}$, $48 \mathrm{~h}$ và $72 \mathrm{~h}$ cho hai lớp thử nghiệm có dùng tham số hóa đối lưu ở miền tính $5 \mathrm{~km}$ (CPS) và không sử dụng (noCPS). Hình d là giá trị trung bình TS úng với các thử nghiệm sủi dụng đối lưu BMJ (BMJ$M E A N)$ và sử dụng $K F(K F-M E A N)$
Minh họa chi tiết hơn về sự khác biệt giữa CPS và noCPS được đưa ra trong hình 3 cho thấy ở hạn dự báo $24 \mathrm{~h}$, việc không sử dụng CPS cho phép giảm sai số rõ rệt ở khu vực Đồng Bằng Bắc Bộ, tuy nhiên ngược lại noCPS dự báo khá khống trên khu vực Đông Bắc. Ở hạn dự báo 48h, cả hai trường hợp đều không thể nắm bắt được mưa rất lớn xảy ra ở khu vực Đông Bắc do vùng thấp được dự báo lệch khá nhiều ra Vịnh Bắc Bộ, tuy nhiên ở hạn $48 \mathrm{~h}$ các thử nghiệm noCPS tiếp tục cho phép giảm dự báo không ở các vùng còn lại một cách rõ rệt (Hình 4 , hàng b). Ở hạn dự báo 72h, hai điểm mưa chính liên quan đến khu vực Móng Cái và Hải Phòng, các 
thử nghiệm noCPS cho phép thể hiện tốt tâm mưa khu vực Hải Phòng so với thử nghiệm CPS. Ở hạn dự báo này, khu vực phía Tây Bắc được dự báo tốt hợp trong noCPS so với CPS (Hình 4 , hàng $\mathrm{c}$ ). Phân tích tổng thể thêm đối với dự báo mưa tích lũy 72 h (Hình 4 , hàng $\mathrm{d}$ ) cho thấy vùng mưa lớn khu vực phía Đông Bắc đã được mở rộng hơn trong dự báo noCPS so với CPS và giảm được sai số một cách rõ rệt ở khu vực Đồng Bằng Bắc Bộ.
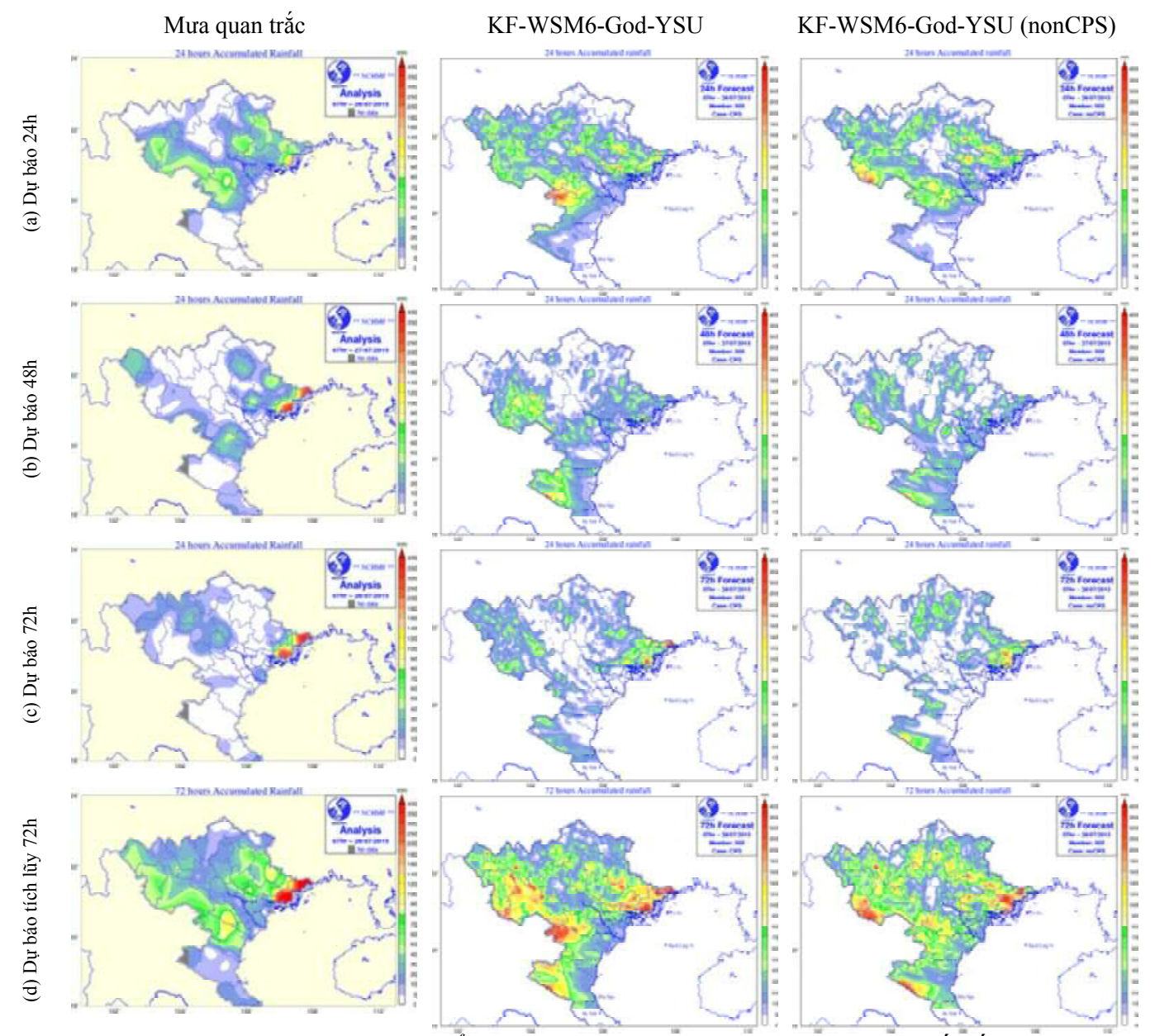

Hình 3. Minh họa dụ báo tại thời điểm dụ báo 00UTC 25/07/2015 với một số cấu hình khác nhau (xem thêm bảng 1) trong đó cột hình bên trái là mura quan trắc, cột hình giũ̃a là có sử dụng tham số hóa, cột hình bên phải là không sủ dụng tham số hóa, hàng (a), (b) và (c) tuoong ứng là dụ báo hạn $24 h$, $48 h$ và $72 h$ cho mưa tích lũy $24 h$, hàng (d) là dụ báo mưa tích lũy $72 h$.

\section{Kết luận}

Nghiên cứu đã thực hiện dự báo đợt mưa lớn kỉ lục trên khu vực Bắc Bộ tháng 7 năm 2015 bằng mô hình WRF-ARW với điều kiện biên từ mô hình toàn cầu GFS thông qua việc hạ quy mô động lực với thiết lập hai lưới tính $15 \mathrm{~km}$ và 5 $\mathrm{km}$ dự báo đến hạn $72 \mathrm{~h}$. Hai thử nghiệm (với 32 cấu hình vật lý khác nhau) đã được thực hiện gồm có sử dụng (CPS) và không sử dụng tham số hóa đối lưu (noCPS) trên lưới tính phân giải cao $5 \mathrm{~km}$. Các kết quả đánh giá cho thấy ở hạn dự báo $24 \mathrm{~h}$, việc sử dụng các sơ đồ tham số hóa cho kết quả tốt hơn so với việc không sử dụng tham số hóa đối lưu trên lưới tính phân giải cao $5 \mathrm{~km}$. Tuy nhiên ở các hạn $48 \mathrm{~h}$ và $72 \mathrm{~h}$, khi không sử dụng tham số hóa đối lưu hay quá trình đối lưu được giải một cách tường mình trong mô hình (explicit) cho phép tăng được kĩ năng dự báo hơn khi thử nghiệm dự báo mưa lớn cho đợt mưa lớn trên khu vực Bắc Bộ. Việc thực hiện với 


\title{
BÀI BÁO KHOA HỌC
}

rất nhiều cấu hình vật lý khác nhau (32 cấu hình) cho phép đánh giá được tổng thể hơn khi không sử dụng CPS ở phân giải cao và có kết quả tương đồng với nhau, ít phụ thuộc vào các cấu hình khác nhau của mô hình thử nghiệm. Vấn đề chưa giảm được sai số ở hạn dự báo 24h đối với thử nghiệm không sử dụng tham số hóa sẽ được tiếp tục cải thiện thông qua quá trình đồng hóa số liệu và sẽ được đề cập đến trong các công trình tiếp theo.

Lời cảm ơn: Các nội dung nghiên cứu trên nằm trong khuôn khổ của đề tài KC.08.06/16-20 "Nghiên cứu xây dựng hệ thống nghiệp vụ dự báo mưa lớn cho khu vực Bắc Bộ Việt Nam", thuộc Chương trình KC.08/16-20 "Nghiên cứu khoa học và công nghệ phục vụ bảo vệ môi trường và phòng tránh thiên tai”.

\section{Tài liệu tham khảo}

1. Hoàng Đức Cường cùng cộng sự, (2011), Nghiên cứu ứng dụng mô hình WRF dụ báo thời tiết và bão ở Việt Nam, Báo cáo tổng kết đề tài NCKH cấp Bộ TN\&MT.

2. Võ Văn Hòa và cộng tác viên, (2010), Nghiên cưu phát triển hệ thống dư báo tổ hợp thời tiết hạn ngắn cho khu vưc Việt Nam, Thuyết minh đăng ký đề tài NCKH cấp Bộ, 17 tr.

3. Walser, A., Lüthi, D. and Schär, C. (2004), Predictability of Precipitation in a Cloud-Resolving Model, Mon. Wea. Rev., 132, 560-577.

4. Gilliland, E. and Rowe, C., (2007), A comparison of cumulus parameterization scheme in the WRF model, 21st conference on Hydrology.

5. Skamarock, W.C., Klemp, J. B., Dudhia, J., Gill, D.O., Barker, D.M., Duda, M.G., Huang, X.Y., Wang, W. and Powers, J.G. (2005), A description of the Advanced Research WRF Version 3, NCAR Tech, Note NCAR/TN-475+STR, 113 pp.

6. Michalakes, J., Dudhia, J., Gill, D., Henderson, T., Klemp, J., Skamarock, W. and Wang, W. (2005), The Weather Research and Forecast Model: Software Architecture and Performance, Proceedings of the Eleventh ECMWF Workshop on the Use of High Performance Computing in Meteorology, World Scientific, pp. 156-168.

\section{EVALUATION ON THE USE OF CUMULUS PARAMETERIZATION SCHEMES FOR HEAVY RAIN FORECAST IN JULY 2015 OVER THE NORTH OF VIETNAM BY HIGH RESOLUTION REGIONAL MODEL}

\author{
Du Duc Tien ${ }^{1}$, Hoang Duc Cuong ${ }^{1}$, Mai Khanh Hung' ${ }^{1}$ Hoang Phuc Lam ${ }^{1}$ \\ ${ }^{1}$ National center of hydro-meteorological forecasting
}

\begin{abstract}
The paper presents the forecasting experiments for the record heavy rainfall in the Northern region in July 2015 by using the WRF-ARW model with boundary conditions from the global model GFS (NCEP, USA). The experiments using two-nested grid at $15 \mathrm{~km}$ and $5 \mathrm{~km}$ and with forecast ranges of 72 hours. Two tests were performed including use and non-use of cumulus parameterization schemes (CPS) on a the finest grid (5 $\mathrm{km}$ domain). The evaluation results show that in the 24-hour forecast ranges, the use of CPS gives better results than not using CPS (noCPS) on a $5 \mathrm{~km}$ resolution grid. However, in the $48 \mathrm{~h}$ and $72 \mathrm{~h}$ forecast ranges and at higher warning thresholds $(50 \mathrm{~mm} / 24 \mathrm{~h}$ and $100 \mathrm{~mm} / 24 \mathrm{~h})$, it is possible to increase the prediction skill with noCPS for the heavy rain in the Northern part of Vietnam.
\end{abstract}

Keyword: Cumulus parameterization scheme, WRF-ARW model, heavy rainfall forecast for the northern part of Vietnam. 